\title{
Um Estudo de caso com o Protótipo de Estimação de Localização baseado em Sistema Multiagente para a melhoria de segurança
}

\author{
Ana Régia de M. Neves ${ }^{1}$, Letícia T. Maia ${ }^{1}$, Célia G. Rallha², Ricardo P. Jacobi² \\ ${ }^{1}$ Departamento de Engenharia Elétrica - Faculdade de Tecnologia - Universidade de \\ Brasília (UnB) \\ Caixa Postal 4521 - 70904-970 - Brasília - DF - Brasil \\ ${ }^{2}$ Instituto de Ciências Exatas - Departamento de Ciências da Computação - \\ Universidade de Brasília (UnB) \\ Caixa Postal 4466 - 70910-900 - Brasília - DF - Brasil \\ \{regianeves, letmaia\}@unb.br, \{ghedini,rjacobi\}@cic.unb.br

\begin{abstract}
Among the variables used to the development of context-aware applications, identification and location are known as primary context because they help the system to provide relevant information to the user. In this article, a prototype based on multiagent system for user identification and location at an indoor environment is presented. Furthermore, a simulation was performed in order to validate the developed prototype.
\end{abstract}

Resumo. Dentre as variáveis utilizadas para o desenvolvimento de aplicações sensíveis ao contexto a identificação e a localização são conhecidas como contexto primário, pois auxiliam o sistema a prover informações relevantes para o usuário. Neste artigo é apresentado um protótipo para identificação e localização de usuários em um ambiente fechado baseado em sistema multiagente. Além disso, foi feita uma simulação a fim de validar o protótipo desenvolvido.

\section{Introdução}

As aplicações sensíveis ao contexto utilizam informações que caracterizam a situação corrente do usuário para fornecer serviços adaptados na realização de suas tarefas [Dey 2001]. Dentre estas, a identificação e a localização são importantes para entender o contexto do usuário móvel.

Este artigo apresenta uma simulação baseada em sistema multiagente para identificar e localizar os usuários dos laboratórios do SG-11 da Universidade de Brasília. Para isso, foi utilizada a plataforma Java Agent DEvelopment Framework $(J A D E)$ que é um software livre distribuído sobre a licença LGPL e implementado em Java. O principal objetivo desta ferramenta é simplificar e facilitar o desenvolvimento de agentes provendo a comunicação, recursos para gerenciar o ciclo de vida e monitorar os agentes em execução [JADE 2003]. 
O artigo está organizado em cinco seções, desconsiderando-se a seção de introdução. A seção 2 aborda aspectos teóricos sobre a computação sensível ao contexto. A seção 3 apresenta uma breve definição de sistema multiagente. Na seção 4 são descritos os trabalhos correlatos. A seção 5 exibe a implementação da proposta e na seção 6 são apresentadas as conclusões e trabalhos futuros.

\section{Computação Sensível ao Contexto}

Refere-se a aplicações que são capazes de monitorar e utilizar dinamicamente informações que provêm do ambiente ou do usuário [Coutaz et al 2005].

De acordo com Dey [2001], contexto é toda informação que pode ser usada para caracterizar a situação de uma entidade (pessoa, lugar ou objeto) que é considerada relevante para a interação entre um usuário e uma aplicação.

Os atributos que definem um contexto podem ser divididos em dois grupos e são dependentes do domínio da aplicação[Castro e Favela 2005]: fatores humanos (hábitos, estado emocional, ambiente social e tarefas realizadas) e propriedades do ambiente físico (localização, infraestrutura e condições físicas).

O processo de desenvolvimento de uma aplicação sensível ao contexto envolve basicamente três etapas [Dey 2001]: i) o contexto deve ser capturado como um conjunto de dados de baixo nível a partir de diferentes sensores; ii) esse conjunto deve ser interpretado para construir uma informação contextual de alto nivel mais significativa para aplicação, por exemplo, ele pode mapear coordenadas GPS (Global Positioning Systems) para um endereço de rua; e iii) deve levar a informação interpretada para a aplicação que é utilizada com outros dados para oferecer e adaptar a computação e serviços.

\section{Sistema Multiagente}

Um Sistema Multiagente (SMA) é um sistema computacional em que dois ou mais agentes interagem ou trabalham em conjunto de forma a desempenhar determinadas tarefas ou satisfazer um conjunto de objetivos [Lesser e Victor 1999].

A área de SMA estuda o comportamento de um grupo organizado de agentes autônomos que cooperam na resolução de problemas que estão além das capacidades de resolução de cada um individualmente [Briot e Demazeau 2001].

Segundo Wooldridge [2002], para um agente ser considerado inteligente, ele deve ser autônomo, apresentar capacidade de comunicação, de cooperação, de raciocínio, deve ter mecanismos de planejamento para executar suas ações e ser adaptável quanto a mudanças ocorridas no ambiente.

O ambiente onde os agentes estão situados é uma parte essencial do sistema [Weyns e Holvoet 2004], por isso, definir suas propriedades é uma decisão de projeto que depende dos requisitos do domínio do problema. Os agentes precisam agir e perceber esse ambiente. Essa percepção pode ser limitada pela capacidade do próprio agente e pelas propriedades do ambiente.

Essa abordagem de SMA pode reduzir o custo e a complexidade associados ao desenvolvimento de sistemas distribuídos, além de permitir uma maior flexibilidade no 
processo de descoberta de soluções de problemas onde essas não podem ser previamente estabelecidas [Jennings et al 1998].

Conforme Bellifemine et al [2007] e Juchem e Bastos [2001], os principais desafios para desenvolver um sistema multiagente são: a) comunicação, definir como ela será realizada entre os agentes e que tipo de protocolo utilizar; b) interação, especificar como essa interação ocorrerá e que linguagens os agentes devem usar para interagirem entre si e combinarem seus esforços; c) coordenação, como garantir essa coordenação entre os agentes para que haja uma coerência na solução do problema ou objetivo que estão tentando alcançar.

\section{Trabalhos Correlatos}

Em Sachima et al [2004] foi desenvolvido um middleware para localização baseado em agentes. Foram definidos quatro tipos de agentes: i) agente de serviço (service provider) que atua como proxy e informa sobre as capacidades e restrições da localização física para fornecer o serviço; ii) agente pessoal (service requester) que solicita a execução de algum serviço em nome do usuário; iii) agente de localização (location-aware middle agent) que compara os agentes de serviço e os agentes pessoais através da localização, capacidade e solicitação para iniciar a comunicação; e iv) agente de dispositivo (device wrapper agent) que atua como proxy de um dispositivo e fornece informações derivadas de sensores para o agente de localização. Toda a comunicação entre os agentes é baseada na linguagem ACL (Agent Communication Language) proposta pela The Foundation of Intelligent Physical Agents - FIPA.

Em Nguyen et al [2008] foi determinada uma comunidade virtual baseada em sistema multiagente para apoiar a descoberta de serviços e localização de usuários em ambientes pervasivos. Foram definidos cinco tipos de agentes: i) agente usuário que solicita sua posição corrente; ii) agente de localização que coleta a posição do usuário por GPS, sensores ou por intensidade de sinal; iii) agente da comunidade que permite ou não a entrada de um agente usuário; iv) agente de serviços que disponibiliza os serviços referentes à comunidade; e v) agente de contexto que armazena dados personalizados sobre o usuário. Para construção do protótipo foi utilizada a plataforma JADE.

Em Neves et al [2010] foi desenvolvido um protótipo baseado em sistema multiagente para estimar a localização de usuários em ambientes fechados. Foram definidos três agentes reativos: i) agente cliente que solicita a sua posição no ambiente; ii) agente mapeamento que procura quais agentes sensores estão disponíveis no ambiente para encontrar a localização do usuário; e iii)agente sensor que procura e encontra o usuário no ambiente. Para a construção do protótipo foi utilizada a plataforma JADE.

\section{A Arquitetura Multiagente e o Protótipo do Sistema de Identificação}

Para identificar e estimar a localização dos usuários dos laboratórios do SG-11 da Universidade de Brasília foi proposta uma arquitetura baseada em sistema multiagentes.

Foram definidos três agentes: cliente, ambiente e sensor. Os agentes cliente e sensor são reativos; e o agente ambiente é reativo com regra de estados. 
A Tabela 1 exibe as percepções, ações e o ambiente para cada agente definido neste trabalho.

Tabela 1. Classificação dos Agentes

\begin{tabular}{|c|c|c|c|}
\hline Agente & Percepção & Ação & Ambiente \\
\hline Cliente & $\begin{array}{l}\text { - Receber } \\
\text { informação de } \\
\text { localização } \\
\text { proveniente do } \\
\text { agente } \\
\text { ambiente } \\
\text { - Receber a } \\
\text { informação de } \\
\text { localização de } \\
\text { outro usuário } \\
\text { proveniente do } \\
\text { agente } \\
\text { ambiente }\end{array}$ & $\begin{array}{l}\text { - Solicitar } \\
\text { localização } \\
\text { para o agente } \\
\text { ambiente } \\
\text { - Solicitar a } \\
\text { localização de } \\
\text { outro usuário } \\
\text { para o agente } \\
\text { ambiente }\end{array}$ & \multirow{3}{*}{$\begin{array}{c}\text { Acessível } \\
\text { Determinístico } \\
\text { Estático } \\
\text { Não-episódico } \\
\text { Discreto }\end{array}$} \\
\hline Ambiente & $\begin{array}{l}\text { - Receber } \\
\text { solicitação do } \\
\text { agente cliente } \\
\text { - Receber a } \\
\text { localização do } \\
\text { usuário } \\
\text { proveniente do } \\
\text { agente sensor } \\
\text { - Armazenar } \\
\text { posição do } \\
\text { usuário no log }\end{array}$ & $\begin{array}{l}\text { - Solicitar a } \\
\text { localização do } \\
\text { usuário para o } \\
\text { agente sensor } \\
\text { - Informar ao } \\
\text { agente cliente } \\
\text { a localização } \\
\text { - Informar ao } \\
\text { agente cliente } \\
\text { a localização } \\
\text { de outro } \\
\text { usuário }\end{array}$ & \\
\hline Sensor & $\begin{array}{l}\text { - Receber a } \\
\text { solicitação do } \\
\text { agente } \\
\text { ambiente }\end{array}$ & $\begin{array}{l}\text { - Localizar } \\
\text { usuário } \\
\text { - Informar ao } \\
\text { agente } \\
\text { ambiente a } \\
\text { localização } \\
\text { requisitada }\end{array}$ & \\
\hline
\end{tabular}


O ambiente simulado é o prédio do departamento de engenharia elétrica da Universidade de Brasília que contém vários laboratórios, o SG11. Todos os docentes, os discentes e os funcionários desse prédio ao possuírem vínculo com a instituição possuem um número de matrícula que servirá para a identificação no ambiente em estudo. Isso é feito para controlar e restringir o acesso a esses laboratórios. Para simulação, o ambiente foi reduzido a três salas (sala 1, sala 2 e sala 3), nas quais o usuário pode se movimentar livremente [Neves et al 2010]. A Figura 1 ilustra o ambiente utilizado.

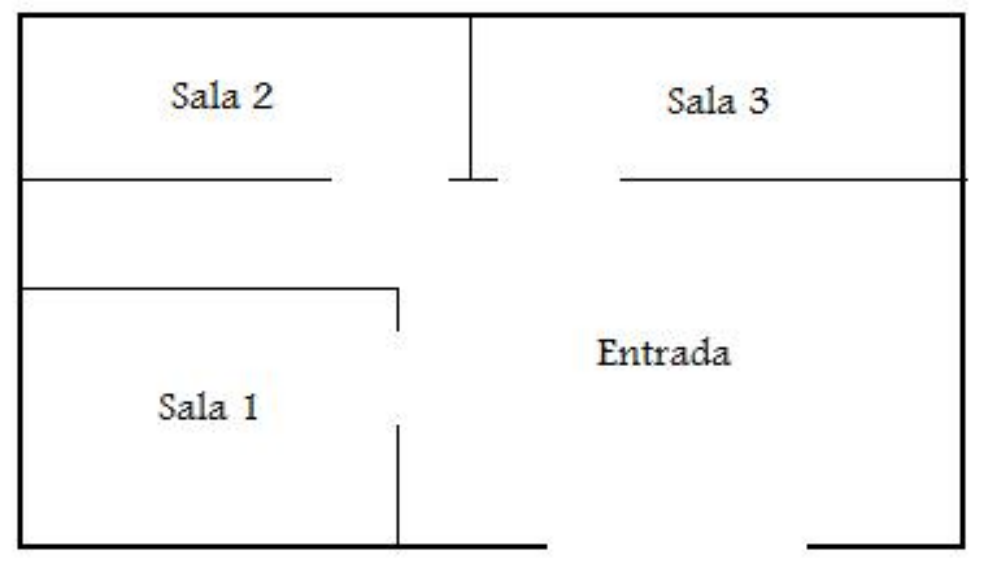

Figura 1. Ambiente Interno.

A arquitetura proposta é exibida na Figura 2, onde o agente cliente está divido em três categorias: i) agente cliente $p$ composto por docentes e podendo variar de 1 a $n$ docentes presentes no prédio; ii) agente cliente $a$ composto por discentes e podendo variar de 1 a $x$ discentes presentes; e iii) agente cliente $f$ composto por funcionários e podendo variar de 1 a $z$ funcionários presentes.

Os agentes clientes podem solicitar a sua própria localização ou a localizaçao de outro agente cliente ao agente ambiente e aguardam a resposta. $\mathrm{O}$ agente ambiente serve como interlocutor entre os agentes clientes e os agentes sensores; e inicia a busca de quais agentes sensores estão disponíveis para localizar os agentes clientes. Cada sala do ambiente contém um agente sensor. Os agentes sensores procuram e localizam o usuários no prédio SG11, depois, repassam a resposta ao agente ambiente o qual informa aos agentes clientes as localizações solicitadas.

Quando é solicitada a localização de um usuário no prédio, o agente ambiente aguarda a resposta dos agentes sensores; caso não seja encontrado, o agente ambiente verifica no $\log$ se há algum registro (data, horário e local mais recente) do usuário solicitado. Apenas usuários cadastrados poderão solicitar a localização de outro. 


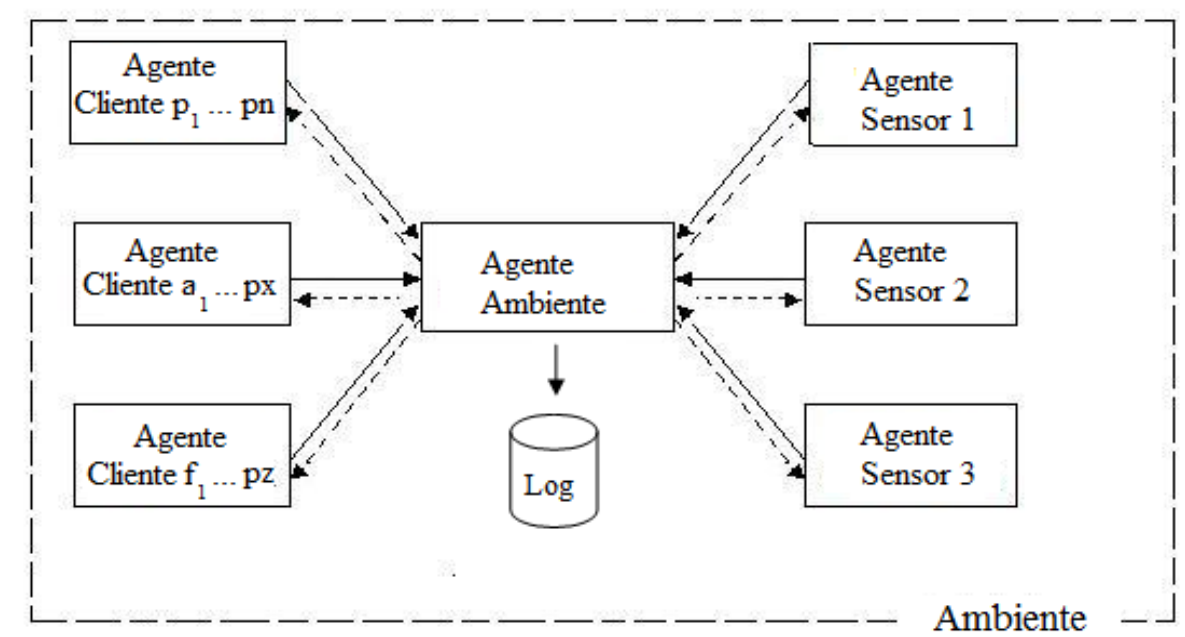

Figura 2. Arquitetura do Sistema de Identificação.

Além disso, foram implementados oito comportamentos que são responsáveis pelas principais ações dos agentes como recepção de mensagens e procura do usuário no ambiente. Esses comportamentos são estendidos da classe behaviours que o JADE oferece. A classe TickerBehaviour permite que esse comportamento seja executado em intervalos de tempo constantes. A classe CyclicBehaviour modela comportamentos atômicos que sempre são executados. A Tabela 2 apresenta a descrição dos comportamentos de cada agente. 
Tabela 2. Comportamento dos Agentes

\begin{tabular}{|c|l|}
\hline Agente & \multicolumn{1}{c|}{ Comportamentos } \\
\hline Cliente & $\begin{array}{l}\text { Procura agente ambiente: solicita ao agente ambiente que informe a } \\
\text { posição no SG11. É um comportamento que estende a classe } \\
\text { TickerBehaviour. } \\
\text { Aguarda retorno de localização: recebe a informação de localização } \\
\text { enviada pelo agente ambiente. É um comportamento que estende a } \\
\text { classe CyclicBehaviour. }\end{array}$ \\
\hline \multirow{5}{*}{ Ambiente } & $\begin{array}{l}\text { Aguarda requisição de usuário: aguarda requisiçães do usuário. É } \\
\text { um comportamento que estende a classe CyclicBehaviour. }\end{array}$ \\
$\begin{array}{l}\text { Procura agente sensor: procura os agentes sensores ativos e solicita } \\
\text { que informe a posição do usuário. É um comportamento que estende } \\
\text { a classe TickerBehaviour. }\end{array}$ \\
$\begin{array}{l}\text { Guarda no log: todos os usuários que entrarem no prédio terão um } \\
\text { registro com hora, data e local que estiveram. É um comportamento } \\
\text { que estende a classe CyclicBehaviour. } \\
\text { Aguarda retorno do agente sensor: recebe a resposta do agente } \\
\text { sensor com a posição do usuário. É um comportamento que estende } \\
\text { a classe CyclicBehaviour. }\end{array}$ \\
\hline \multirow{5}{*}{ Sensor } & $\begin{array}{l}\text { Aguarda requisição do agente ambiente: aguarda requisiçães de } \\
\text { localização do agente ambiente e responde com a posição do } \\
\text { usuário. É um comportamento que estende a classe } \\
\text { CyclicBehaviour. } \\
\text { Detecta movimento usuário: detecta a movimentação do usuário. É } \\
\text { um comportamento que estende a classe TickerBehaviour }\end{array}$ \\
\hline
\end{tabular}

A Figura 3 mostra um cenário que envolve a interação dos agentes no ambiente, com os seguintes passos: (1) o agente cliente solicita sua localização ao entrar no ambiente ou a localização de um outro usuário; (2) o agente ambiente recebe a solicitação do agente cliente, o identifica com um ID de matrícula, procura quais agentes sensores estão disponíveis com o serviço de localização e repassa a solicitação do agente cliente com o ID de matrícula aos agentes sensores; (3) os agentes sensores recebem a mensagem do agente ambiente com o ID do usuário, procuram o usuário e informam a localização solicitada ao agente ambiente; (4) o agente ambiente guarda todas as informações de localização no log, registrando a hora, data e sala que o usuário está; (5) o agente ambiente envia a localização solicitada ao agente cliente. 


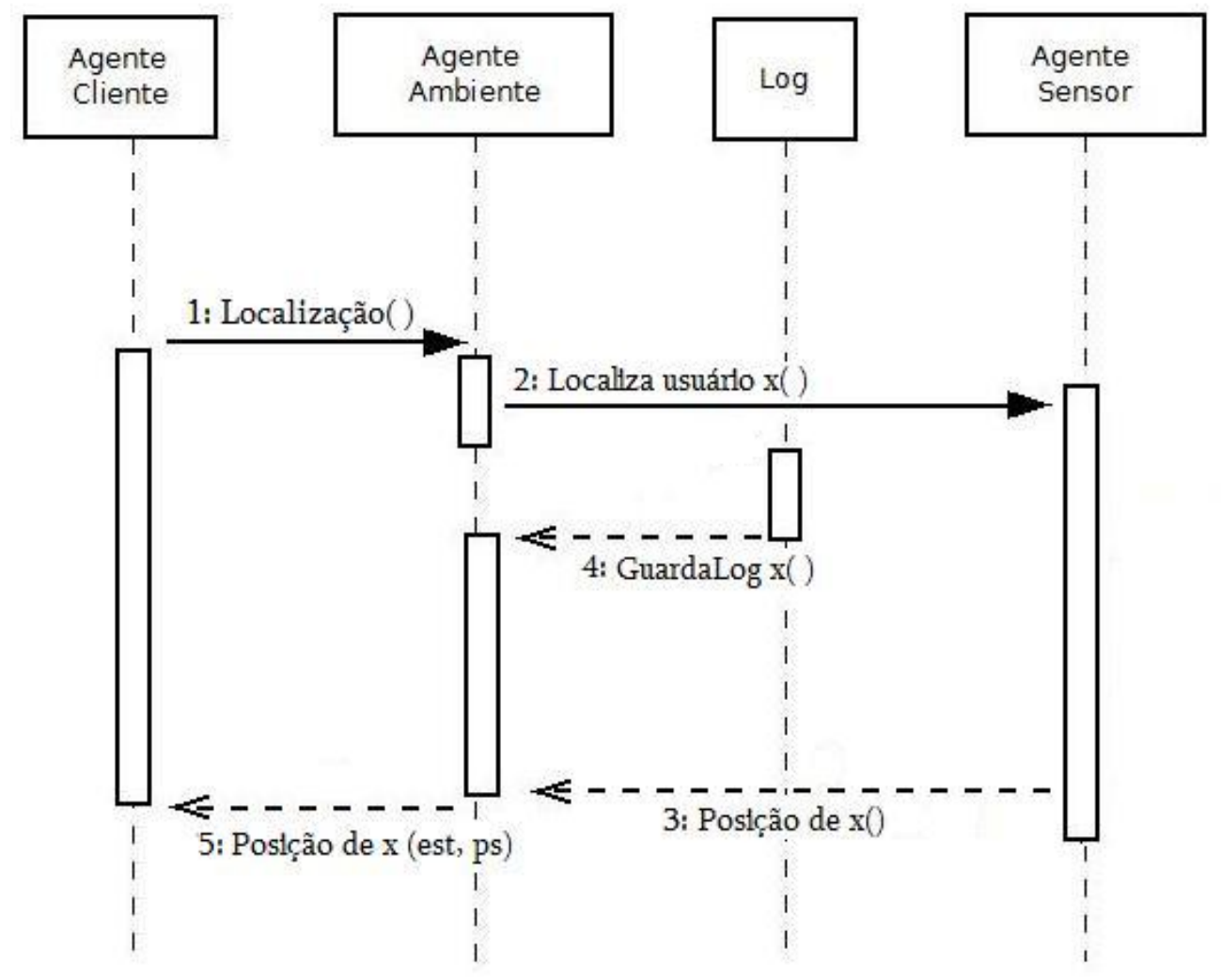

Figura 3. Diagrama de Sequência para a Localização do Usuário na simulação.

Assim, a partir do momento que o usuário for identificado com o ID, sua movimentação no ambiente será rastreada.

A Figura 4 apresenta um screenshot do console com um agente cliente chamado Rosinha. Esta usuária é identificada com uma ID (robo 3) a fim de ser monitorada no ambiente. As localizações (sala 1, sala 2, sala 3) da usuária são definidas em intervalos de tempos aleatórios no ambiente. 


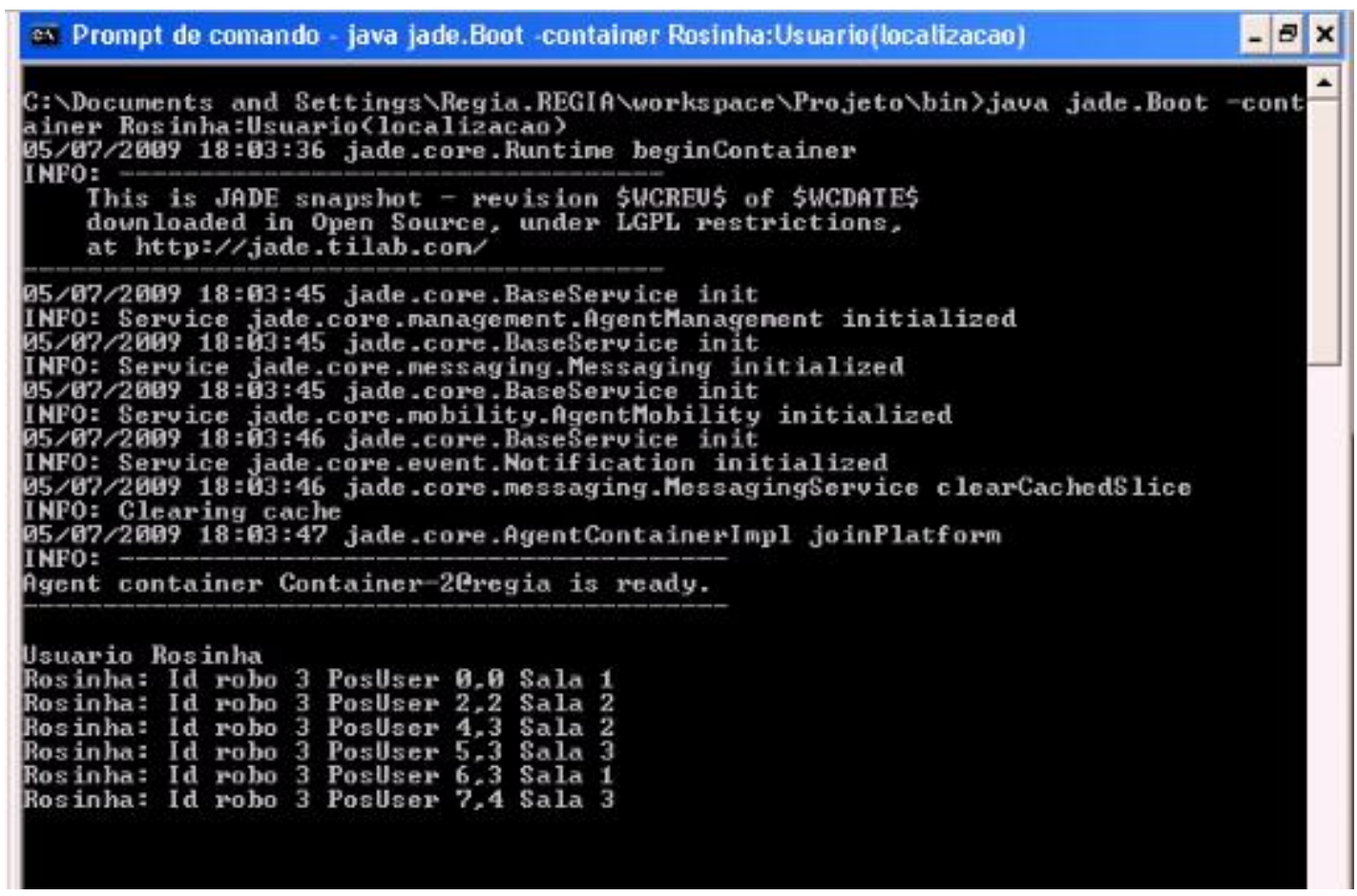

Figura 4. Screenshot do Console das Posições do Usuário.

\section{Conclusões e Trabalhos Futuros}

Como visto, estimar a localização de um usuário num ambiente é um dos aspectos mais importantes para sistemas sensíveis ao contexto, pois, o sistema poderá fornecer serviços de acordo com esta característica e conservar seguro esses serviços e as pessoas que os utiliza.

Baseado nisso, foi realizada uma simulação para identificação e localização de docentes, discentes e funcionários de três laboratórios do departamento de Engenharia Elétrica da universidade de Brasília utilizando sistema multiagente através da plataforma JADE.

Essa abordagem foi escolhida devido à abstração fornecida para a concepção de sistemas complexos, onde deve ocorrer a junção de diferentes domínios de conhecimento para a solução do problema, como, em ambientes sensíveis ao contexto. Além disso, se mostrou adequada ao desenvolvimento do protótipo proposto num ambiente estático, facilitando a definição de diversos comportamentos dos agentes.

Assim, planeja-se implementar uma arquitetura mais robusta e complexa no ambiente real, desenvolver agentes cognitivos para maior autonomia de decisão e proatividade dos agentes no sistema, além de utilizar outras técnicas de Inteligência Artificial, como, redes neurais artificiais, para detecção mais precisa da posição e uso de ontologias para contextualização de ambientes e serviços, conforme grupos de usuários e cenários distintos.

Uma das limitações da implementação de uma arquitetura multiagente no ambiente real refere-se ao acréscimo de novos agentes, pois, mesmo que aumente a funcionalidade do sistema, a coordenação dos diferentes tipos de agentes, cada um com certo tipo de autonomia, fica mais complexa. 


\section{Referências}

Abowd, G. D. e Mynatt, E. D. (2000), Charting past, present, and future research in ubiquitous computing. ACM Transactions on Computer-Human Interaction, 7(1): 29-58.

Bellifemine, F. L.; Giovanni, C.; Greenwood, D. (2007), Developing Multi-Agent Systems with JADE. John Wiley \& Sons, Ltd.

Briot, J.-P. e Demazeau, Y., editors (2002). Principes et architecture des systèmes multi-agents. Hermes, Paris.

Castro, L. A. e Favela, J. (2005) "Continuous Tracking of User Location in WLANs Using Recurrent Neural Networks", In Proc. Sixth Mexican International Conference on Computer Science. (ENC'05).

Coutaz, J.; Crowley, J.; Dobson, S.; Garlan, D. (2005), Context is key, Communications of the ACM, Vol 48, No 3, pp. 49-53.

Dey, A. K. (2001), Understanding and Using Context. Personal and Ubiquitous Computing Springer-Verlag, London, UK; Vol. 5, No 1, 201, pp. 4-7.

Jade. JADE (2003) - Java Agent Development Framework. Disponível em http://jade.tilab.com/, Junho.

Jennings, N. R. (2001) “An Agent-based Approach for Building Complex Software Systems", Communications of the ACM archive, vol. 44, pp.35-41.

Juchem, M. e Bastos, R. M. (2001), Engenharia de Sistemas Multiagentes: Uma investigação sobre o estado da arte. Technical Report Series, No 014.

Lesser, Victor (1999) Cooperative Multi-Agent Systems: A Personal View of the State of the Art, IEEE Transactions on Knowledge and Data Engineering, Vol. 11, No1,.

Loke, S. (2007), Context-Aware Pervasive Systems: architectures for a new breed of applications. Auerbach Publications.

Neves, A. R. M; Maia, L. T.; Ralha, C. G.; Jacobi, R. P. (2010) "Prototype for Indoor Localization Based on Multiagent System", In Third International Conference on Intelligent \& Advanced System.

Nicholas R. Jennings; Sycara, K. e Wooldridge, M. (1998). A roadmap of agent research and development. Journal of Autonomous Agents and Multi-Agent Systems, $1(1): 7-38$

Nguyen, T; Loke, S.; Torabi T.; Lu, H. (2008) "Multiagent Place-Based Virtual Communities for Pervasive Computing", In Sixth Annual IEEE International Conference on Pervasive and Communications.

Orr, R. J e Abowd G. D. (2000) "The Smart Floor: A mechanism for natural user identification and tracking", In Proc. Conference on Human Factors in Computing Systems, ACM Press, New York.

Russell, S.; Norvig, P. (2003) “Artificial Intelligence: A Modern Approach”, Prentice Hall, Second Edition. 
Sashima, A.; Izumi, N.; Kurumatani, K. (2004) "Location-aware Middle Agents in Pervasive Computing", In The 2004 International Conference on Pervasive Computing and Communications, 2004, pp. 820-826.

Torsun, I. S. (1995), Foundations of Intelligent Knowledge-based systems. London: Academic Press.

Want, R.; Hoopper, A.; Falcão, V.; Gibbons, J. (1992) "The Active Badge Location System”. ACM Trans. On Information Systems, 10 (1): 91-102.

Weyns, D.; Holvoet, T. (2004) “A Formal Model for Situated Multiagent Systems", Fundamenta Informaticae, v.63 (2-3), pp.125-158.

Wooldridge, M. (2002) “An Introduction to Multiagent Systems”. Ed. John Wiley \& Sons Ltd., 2 ed. 